\title{
News internet
}

\author{
Valérie Duflot ${ }^{*}$
}

\section{Agenda}

\section{9th Workshop on Fats and Oils as Renewable Feedstock for the Chemical Industry: Karlsruhe, Germany, March 19-21, 2017}

The environmentally sound and sustainable use of natural resources is an important worldwide challenge. At present, fats and oils are the most widely used renewable raw materials in the chemical industry, since they offer widespread possibilities for different applications. The Workshop on "Fats and Oils as Renewable Feedstock for the Chemical Industry" has evolved to a major global oleochemical meeting, attracting participants from all over the world. Important contributions to the workshop have been documented in special issues of European Journal of Lipid Science and Technology. The 9th Workshop "Fats and Oils as Renewable Feedstock for the Chemical Industry" will discuss new developments as well as future perspectives of the chemical usage of fats and oils including terpenes. Presentations from industry representatives will show the progress in introducing renewable materials to the market.

The workshop in Karlsruhe (Germany, March 19-21, 2017) will cover the following topics (amongst others):

- chemistry of fats and oils and other renewable resources;

- synthesis of fine chemicals;

- monomer/polymer synthesis;

- catalysis;

- sustainability;

- introducing renewable materials to the market.

More information: http://abiosus.org/kit-workshop-2017.html

\section{Publication}

\section{Chiffres clés 2015-2016 des oléagineux}

Terres Univia a publié fin 2016 une nouvelle brochure, les Chiffres clés 2015-2016 des oléagineux, que vous pouvez télécharger directement sur le site de Terres Univia (PDF, $1,7 \mathrm{Mo})$.

\section{Agrément}

\section{Analyses physicochimiques et sensorielles des huiles d'olive à I'ITERG}

L'ITERG a obtenu le renouvellement de l'agrément du Conseil oléicole international (COI) pour la période du $1^{\text {er }}$ décembre 2016 au 30 novembre 2017 sur les analyses physicochimiques et sensorielles des huiles d'olive.

Les listes des laboratoires agréés sont disponibles sur le site du COI.

\section{Inauguration}

\section{Cirad : la Maison de la télédétection double sa surface}

La Maison de la télédétection (MTD), à Montpellier, a inauguré ses nouveaux locaux lundi 10 octobre 2016. Avec une surface doublée et de nouvelles infrastructures de calcul et de réception d'images, la MTD œuvre en faveur d'une recherche renforcée dans les domaines de l'environnement, l'agriculture et d'aménagement territorial, en France et dans les pays du Sud.

Implantée à Montpellier depuis plus de 20 ans, la MTD dédie son activité au traitement des données d'observation de la Terre pour la gestion durable de l'agriculture et des territoires. Soutenue par la Région, l'Etat et l'Europe, la MTD s'est récemment dotée de nouvelles installations et équipements pour renforcer ses recherches ainsi que sa stratégie de mutualisation et de mise à disposition de données et de compétences.

La MTD accueille désormais une infrastructure de calcul et de stockage, une antenne de réception satellitaire et une salle de réception d'images dédiées. Également à disposition des chercheurs mais aussi des étudiants et des entreprises innovantes : une salle de conférence, cinq salles de formation, ainsi que de nouveaux bureaux. Ces nouvelles installations permettront de démultiplier les capacités d'activités de la MTD et de contribuer ainsi au développement de la filière de télédétection spatiale française en appui aux politiques publiques et au rayonnement international du site de Montpellier, de l'Occitanie et de la France.

\footnotetext{
*Correspondance : contact@ocl-journal.org
} 


\section{Communication}

\section{L'Esprit Sorcier dédie trois émissions aux protéines végétales}

Trois émissions de L'Esprit Sorcier dédiées aux protéines végétales ont été diffusées du 17 octobre au 5 novembre 2016 sur Campagne TV :

- la place des protéines dans l'alimentation de demain ;

- les légumes secs dans l'assiette ;

- les légumineuses au cœur de l'agriculture de demain.

Elles peuvent toujours être visionnées sur Internet.

\section{Changement climatique}

\section{Étudier les impacts de la concentration atmosphérique en $\mathrm{CO}_{2}$ sur la physiologie des plantes}

Durant des millénaires, les teneurs en dioxyde de carbone $\left(\mathrm{CO}_{2}\right)$ de l'atmosphère sont restées relativement stables : elles variaient entre 200 et 300 parties par million (ppm), sans être influencées outre-mesure par les activités humaines. Les choses ont changé suite à la Révolution industrielle. Depuis un siècle et demi, l'utilisation à grande échelle des énergies fossiles comme le charbon, le gaz ou le pétrole a entrainé un rejet massif de $\mathrm{CO}_{2}$ dans l'atmosphère. Actuellement, le taux de $\mathrm{CO}_{2}$ atmosphérique croît de façon exponentielle ; il a désormais dépassé le seuil de $400 \mathrm{ppm}$, et cette tendance ne semble pas devoir s'infléchir. $\mathrm{Le} \mathrm{CO}_{2}$ étant l'un des principaux gaz à effet de serre, l'augmentation de sa concentration aggrave le changement climatique. Mais ses effets ne s'arrêtent pas là...

Comme le souligne Denis Fabre de l'UMR AGAP / équipe PAM, responsable du plateau d'écophysiologie : «En tant qu'être humain, nous ne remarquons pas l'augmentation actuelle. Et pour cause : à chaque expiration, nous rejetons $50000 \mathrm{ppm}$ de $\mathrm{CO}_{2}$. Dans un bureau non aéré, en fin de journée, les $500 \mathrm{ppm}$ sont donc facilement atteintes. Les plantes, en revanche, sont très sensibles aux variations des taux de $\mathrm{CO}_{2}$. »

Rien d'étonnant, puisque le $\mathrm{CO}_{2}$ est l'un des facteurs environnementaux qui influencent la photosynthèse, le processus grâce auquel les plantes fabriquent, à partir de l'énergie lumineuse, les sucres indispensables à leur fonctionnement. On pourrait supposer que plus les plantes ont de $\mathrm{CO}_{2}$ à disposition, plus elles fabriquent de sucres, disposant ainsi d'un surplus d'énergie pour croître et produire davantage. Malheureusement, les choses ne sont pas si simples.

« En cas d'augmentation du $\mathrm{CO}_{2}$ atmosphérique, certaines plantes accroissent leurs niveaux de photosynthèse et produisent davantage de biomasse, tandis que d'autres stagnent, explique Denis Fabre. Chez le riz par exemple, les variétés du groupe Indica profitent de l'atmosphère enrichie en $\mathrm{CO}_{2}$ pour leur photosynthèse, contrairement aux variétés du groupe Japonica. » En plein champ, les différences de rendement liées à cette fertilisation par le $\mathrm{CO}_{2}$ peuvent varier de 3 à $36 \%$. Cette disparité s'expliquerait notamment par le fait qu'il ne s'agit pas seulement de produire des sucres, encore faut-il pouvoir les exporter depuis les feuilles, puis les stocker...
En effet, quand une plante photosynthétise, elle produit de la matière, qu'elle stocke dans des « organes puits » : dans le cas du riz, il s'agit des panicules ou des talles (les brins secondaires ou tertiaires). Si la capacité de photosynthèse d'un végétal augmente (en raison d'un plus fort taux de $\mathrm{CO}_{2}$ ambiant, par exemple) sans que sa capacité de stockage n'en fasse de même, que se passe-t-il ? «Dans ce cas, la plante est comme rassasiée, indique Denis Fabre. La photosynthèse diminue, et sa croissance stagne. Au Cirad, nos travaux sur le riz visent notamment à évaluer l'impact de la capacité de stockage, et donc des organes puits, sur l'utilisation du $\mathrm{CO}_{2}$ atmosphérique. Nous cherchons aussi à déterminer comment cette capacité de stockage régule la photosynthèse. » Pour parvenir, les écophysiologistes du Cirad se sont récemment équipés de deux phytotrons dernier cri.

Dans ces chambres climatiques high-tech, les plantes sont soumises aux climats du futur, telles que les prédisent les modèles actuels. "Grâce à ces phytotrons, nous pouvons étudier le comportement de plants de riz dans un milieu très contrôlé, sous deux conditions de $\mathrm{CO}_{2}: 400 \mathrm{ppm}$ et $800 \mathrm{ppm}$ (cette dernière valeur correspond au taux de $\mathrm{CO}_{2}$ à l'horizon 2100 , tel que prédit par le scénario intermédiaire RCP6.0 du GIEC). Afin de déterminer l'impact des organes puits comme les panicules sur la photosynthèse, nous les éliminons sur la moitié des plants », explique Denis Fabre.

Les paramètres détaillés de la photosynthèse sont ensuite mesurés grâce à un analyseur bardé de capteurs et d'analyseurs infrarouges. La chambre de mesure de cet outil haut de gamme, longue de quelques centimètres seulement, permet de soumettre une portion de feuille au climat de n'importe quel endroit de la planète. « Nous pouvons y faire varier la température par pas de $0,1^{\circ} \mathrm{C}$, l'humidité, la quantité d'éclairage, sa qualité, et même la vitesse du vent latéral!", s'enthousiasme le chercheur. Outre le niveau de photosynthèse, l'analyseur mesure de nombreux paramètres renseignant sur l'état physiologique de la plante, tels que l'ouverture des stomates (les orifices par lesquelles les plantes transpirent). Il permet aussi d'évaluer son niveau de stress.

Les résultats préliminaires sont prometteurs et confirment l'intérêt d'étudier les impacts de la concentration atmosphérique en $\mathrm{CO}_{2}$ sur la physiologie des plantes. " Quand on parle de changement climatique, on parle souvent de températures, et plus rarement de $\mathrm{CO}_{2}$. Le Cirad se démarque par sa capacité à analyser les effets de la concentration atmosphérique en $\mathrm{CO}_{2}$ sur la photosynthèse, et donc d'évaluer plus en détail le fonctionnement des plantes dans un contexte de changement climatique », conclut l'écophysiologiste.

À terme, ces travaux aideront les sélectionneurs à choisir les variétés profitant au mieux de l'augmentation en $\mathrm{CO}_{2}$ atmosphérique due au changement climatique, tout en résistant à la hausse des températures et au manque d'eau. Ils permettront également d'améliorer les modèles de fonctionnement des plantes. Enfin, ils pourraient aussi servir à améliorer les modèles climatiques. En effet, plus la photosynthèse augmente, plus les stomates des plantes se ferment, ce qui limite les rejets de vapeur d'eau dans l'atmosphère et génère des climats plus secs. Mieux comprendre le fonctionnement de la photosynthèse permettrait donc de prédire plus précisément l'impact des végétaux sur le climat. 


\section{Recherche}

\section{L'ITERG participe au projet OLEUM « Garantir la qualité et l'authenticité de I'huile d'olive »}

Le projet OLEUM « Garantir la qualité et l'authenticité de l'huile d'olive », financé par la Commission européenne dans le cadre de l'appel d'offre H2020, a débuté le $1^{\mathrm{er}}$ septembre 2016 pour une période de quatre ans. Il regroupe une vingtaine de partenaires, dont plusieurs universités européennes (Bologne, Udine, Pérouse, Thessalonique, Barcelone, du Littoral en Slovénie), des instituts de recherche (CSIC, JRC, FERA, ITERG, Eurofins) et des représentants de l'industrie.

L'objectif est d'améliorer l'évaluation de la qualité et de la pureté de l'huile d'olive vierge, par la mise en place de nouveaux outils analytiques, et la prise en main de ces outils par la communauté des laboratoires d'analyse via le réseau OLEUM.

\section{Internet}

\section{Le site d'OLEAD est en ligne}

Découvrez le nouveau site internet d'OLEAD, la plateforme technologique commune des centres techniques de l'ITERG (Institut technique des corps gras) et de Terres Inovia, avec l'appui financier de Sofiprotéol : www.olead.fr.

\section{Agronomie}

\section{Succès des colzas associés aux légumineuses}

Raisonner son implantation avec des colzas associés, c'est augmenter les rendements de $10 \%$, soit un gain moyen de trois quintaux par hectare, voire parfois nettement plus en conditions difficiles. Des observations confirmées chaque année depuis 2011. Les avantages sont nombreux avec notamment une augmentation du potentiel agronomique de la parcelle qui se répercute sur les cultures suivantes. Et des bénéfices environnementaux indéniables: un recours plus limité aux herbicides, aux insecticides et aux engrais. Les charges de désherbage ont ainsi diminué de 20 à $30 \%$ et la technique a permis de supprimer l'utilisation d'au moins un insecticide. La réduction d'apport d'engrais permet d'économiser 30 unités d'azote en moyenne. Cette technique innovante est matériellement accessible, puisque aucun équipement supplémentaire ou spécifique n'est nécessaire.

La technique du colza associé à des légumineuses a fait ses preuves chez les exploitants agricoles qui la maîtrisent. Avec des bénéfices économique, environnemental et agronomique, l'association du colza à une légumineuse est une solution pratique pour «produire plus et mieux ». Les équipes de Terres Inovia testent cette innovation depuis cinq ans sur plus de 160 sites d'expérimentation en France. Trèfle d'Alexandrie, fenugrec, lentille, vesce commune ou pourpre et féverole sont parmi les nombreuses légumineuses pouvant être associées en mélange au colza lors du semis. Elles sont semées en même temps que le colza et accompagnent ce dernier jusqu'au cœur de l'hiver. Le gel faisant généralement disparaître ces plantes compagnes sensibles au gel. Elles auront contribué à le protéger de ravageurs d'automne, comme la grosse altise ou le charançon du bourgeon terminal, et à limiter l'envahissement par les mauvaises herbes. De plus, cette présence ne sera pas neutre pour la suite, par sa contribution à l'alimentation en azote du colza sur l'ensemble du cycle.

Le département de l'Indre, l'un des premiers producteurs de colza, est un véritable modèle pour le développement de cette innovation agronomique. Pascal Amary et Jean-François Renaud, agriculteurs de la région dont les parcelles se situent à $20 \mathrm{~km}$ au Nord-Ouest de Châteauroux, se sont lancés dans le colza associé en 2011. Se limitant au départ à quelques hectares, cette pratique s'étend aujourd'hui sur la quasiintégralité de leurs surfaces de colza. En 2016, dans l'exploitation de Pascal Amary, 78 ha sur 80 sont menés avec la technique innovante. Dans celle de Jean-François Renaud, ce sont 100 ha sur 110.

Les producteurs qui maîtrisent cette technique témoignent de sa performance mais également de la nécessité d'un apprentissage progressif. Pour eux, il ne s'agit pas d'une recette à appliquer. Il y a des principes et des clés de raisonnement à suivre avec l'obligation d'adapter finement ses pratiques à la parcelle et de les ajuster en fonction du climat. Une approche nouvelle qui constitue une porte d'entrée efficace pour faire évoluer les systèmes de production céréaliers avec une double performance - économique et écologique - et les rendre plus résilients aux aléas.

Pour Terres Inovia, cette technique qui concerne plusieurs dizaines de milliers d'hectares doit se développer prioritairement sur plus de 100000 ha situés dans le croissant historique des petites terres à colza, du Poitou-Charentes à la Bourgogne. Certains secteurs de cette zone particulièrement exposés aux insectes d'automne où sévissent des résistances aux insecticides sont les plus concernés. À terme, plus de $40 \%$ de la sole française de colza (1 $500000 \mathrm{ha}$ ) pourrait valoriser pleinement cette technique. Le ministère de l'Agriculture et la profession sont tombés d'accord pour accompagner le développement de cette innovation: elle bénéficie d'un certificat d'économie de produits phytosanitaires (CEPP). L'accompagnement agronomique est essentiel. Ainsi, Terres Inovia a récemment édité un point technique ${ }^{1}$ et propose une formation $^{2}$ spécifique aux techniciens de terrain et aux agriculteurs désireux de tester avec succès cette technique.

\section{Agro-écologie}

\section{Le Cirad et I'Inra affichent leur positionnement commun}

L'agro-écologie constitue un des chantiers scientifiques prioritaires du Cirad et de l'Inra. Au Nord, comme au Sud, la démarche agro-écologique est une voie majeure pour développer une agriculture durable et multi-performante. Elle dessine ainsi un nouveau paradigme pour concevoir des systèmes alimentaires durables. Dans cette optique, le Cirad et l'Inra ont identifié ensemble cinq axes de recherche portant sur la biodiversité, les grands cycles biogéochimiques, les paysages et des territoires, les systèmes de production et les systèmes d'innovation.

\footnotetext{
${ }^{1}$ Colza associé à un couvert de légumineuses gélives, édition Terres Inovia, juin 2016. http://www.terresinovia.fr/publications/editionspayantes/points-techniques/.

${ }^{2}$ Associer son colza à des légumineuses : conduite et bénéfice pour la culture et le système de culture. http://www.terresinovia.fr/formation/ detail/formation/379/.
} 


\section{Prospective}

\section{Huiles et protéines végétales : quatre scénarios pour 2030}

Augmentation de la démographie mondiale, ouverture des marchés, changement climatique, etc. : difficile de prévoir l'avenir dans un contexte mondial où les défis à relever sont nombreux. Pour le préparer au mieux, Terres Inovia a lancé fin 2013 un exercice de prospective à horizon de 15 ans, visant à imaginer ce qui pourrait se passer en 2030. L'objectif ? Apporter aux acteurs de la filière des huiles et protéines végétales des éclairages sous forme de scénarios d'évolution du contexte et des débouchés pour leur permettre d'élaborer des pistes d'actions.

Dix-huit experts issus des entreprises, des organisations interprofessionnelles et des institutions de recherche et de développement se sont réunis à 14 reprises de septembre 2013 à décembre 2015 afin d'élaborer une vision prospective. De cette réflexion sont sortis quatre scénarios qui illustrent les différentes logiques d'évolution du contexte et les enjeux majeurs.

Allant d'un contexte de désorganisation et de tensions à une vraie coopération internationale, les différents scénarios envisagent pour 2030 des situations très différentes avec des impacts variés sur le secteur des huiles et protéines végétales.

Un premier scénario «Vers le chaos » dessine un monde confronté à une crise économique et politique et aux impacts du changement climatique. Cela provoque de fortes tensions alimentaires et des inégalités croissantes. Les protéines animales sont chères, permettant un développement des protéines végétales dans l'alimentation.

Un deuxième scénario «La raison des blocs » laisse une grande place aux politiques régionales et au bilatéralisme. La demande en huiles et protéines végétales est dynamique et diversifiée avec le développement des énergies renouvelables - notamment les biocarburants - des consommations accrues de protéines animales dans les pays émergeants, la montée du végétalisme et de l'alimentation sans OGM en Europe.

Un troisième scénario «Confiance » décrit un monde de faible croissance démographique dans lequel les pays coopèrent pour lutter contre le changement climatique. L'oléochimie et les biocarburants progressent.

Enfin, un quatrième scénario « La rupture climatique » dans lequel le monde doit s'accorder sur des mesures d'économie et une coopération forcée par les tensions climatiques et environnementales. La consommation de protéines animales stagne, voire diminue, dans certaines régions et la consommation de protéines végétales est privilégiée en Europe.

Pour la filière des huiles et protéines végétales, les différents scénarios révèlent des points essentiels. La croissance des productions agricoles restera une priorité à l'horizon 2030. Le changement climatique peut avoir des conséquences détermi- nantes sur ces productions, voire être un élément de rupture. Par ailleurs, la tension pour les protéines est une quasi-certitude. L'importance relative prise par les protéines végétales et la bonne valorisation économique des huiles varient en fonction des scénarios. À noter aussi, l'épuisement des ressources marines qui devrait entraîner un développement de l'aquaculture et des tensions sur les oméga 3. Pour terminer, une bonne nouvelle, l'équilibre du bilan protéique de l'Europe, aujourd' hui très déficitaire, est possible à l'horizon 2030.

La synthèse de l'étude «Prospective huiles et protéines végétales 2030 » est téléchargeable en version PDF (6,1 Mo) sur le site de Terres Inovia.

\section{Un développement prometteur}

Le département de l'Indre, l'un des premiers producteurs de colza, est un véritable modèle pour le développement de cette innovation agronomique. Pascal Amary et Jean-François Renaud, agriculteurs de la région dont les parcelles se situent à $20 \mathrm{~km}$ au Nord-Ouest de Châteauroux, se sont lancés dans le colza associé en 2011. Se limitant au départ à quelques hectares, cette pratique s'étend aujourd' hui sur la quasi-intégralité de leurs surfaces de colza. En 2016, dans l'exploitation de Pascal Amary, 78 ha sur 80 sont menés avec la technique innovante. Dans celle de Jean-François Renaud, ce sont 100 ha sur 110.

Les producteurs qui maîtrisent cette technique témoignent de sa performance mais également de la nécessité d'un apprentissage progressif. Pour eux, il ne s'agit pas d'une recette à appliquer. Il y a des principes et des clés de raisonnement à suivre avec l'obligation d'adapter finement ses pratiques à la parcelle et de les ajuster en fonction du climat. Une approche nouvelle qui constitue une porte d'entrée efficace pour faire évoluer les systèmes de production céréaliers avec une double performance - économique et écologique - et les rendre plus résilients aux aléas.

Pour Terres Inovia, cette technique qui concerne plusieurs dizaines de milliers d'hectares doit se développer prioritairement sur plus de 100000 ha situés dans le croissant historique des petites terres à colza, du Poitou-Charentes à la Bourgogne. Certains secteurs de cette zone particulièrement exposés aux insectes d'automne où sévissent des résistances aux insecticides sont les plus concernés. À terme, plus de $40 \%$ de la sole française de colza (1 $500000 \mathrm{ha}$ ) pourrait valoriser pleinement cette technique. Le ministère de l'Agriculture et la profession sont tombés d'accord pour accompagner le développement de cette innovation: elle bénéficie d'un certificat d'économie de produits phytosanitaires (CEPP). L'accompagnement agronomique est essentiel. Ainsi, Terres Inovia a récemment édité un point technique ${ }^{3}$ et propose une formation $^{4}$ spécifique aux techniciens de terrain et aux agriculteurs désireux de tester avec succès cette technique.

Citation de l'article : Duflot V. 2017. News internet. OCL 2017, 24(1) N101.

\footnotetext{
${ }^{3}$ Colza associé à un couvert de légumineuses gélives, édition Terres Inovia, juin 2016. http://www.terresinovia.fr/publications/editionspayantes/points-techniques/.

${ }^{4}$ Associer son colza à des légumineuses : conduite et bénéfice pour la culture et le système de culture. http://www.terresinovia.fr/formation/ detail/formation/379/.
} 